\title{
A WAVELET BASED RECURSIVE RECONSTRUCTION ALGORITHM FOR LINEAR MEASUREMENTS
}

\author{
Orhan Arıkan \\ Electrical Engin. Dept., Bilkent Univ. 06533, Ankara, Turkey. \\ Tel: +90-312-266-4307, Fax: +90-312-266-4126 \\ e-mail: oarikan@ee.bilkent.edu.tr
}

\begin{abstract}
A recursive algorithm is proposed to obtain an efficient regularized least squares solution to large linear system of equations which arises in many physical measurement models. The algorithm recursively updates the solution in an increasingly larger dimensional subspace whose basis vectors are chosen as a subset of a complete wavelet basis. Robust criterions on how to chose the basis vectors at each iteration, and when to stop the iterations are given.
\end{abstract}

\section{INTRODUCTION}

In many physical measurements, the unknowns are related to the data through a noisy linear transformation. Reconstruction of the unknowns from the data has been the subject matter of many inverse problems arising in a vast class of applications including remote sensing, geophysical signal processing and speech processing. Various inversion algorithms have been developed to provide reliable and accurate reconstructions $[1,2,3]$. In practice, when the signal to noise ratio is high, these approaches provide satisfactory reconstructions that are relatively insensitive to the choice of various parameters involved in the corresponding cost functions. However, in applications where the signal to noise ratio is low, the choice of these parameters becomes a critical issue. In these cases adaptive choice of these parameters becomes a necessity which often increases the amount of computation drastically [4].

A very important first step of the inverse problems is the parameterization of the unknowns. In many applications, where the sensitivity of the measurements varies across the space of the unknowns, the space of the unknowns is par titioned into cells of non-uniform sizes. The dimensions of cells becomes larger when the sensitivity of the measurements to those cells becomes weaker. In order to keep the computational complexity at a low level, usually data independent partitions are used. In this way in the reconstructions a better than average performance is obtained compared with the case where uniform partitions are used. However, since fixed partitions are used, the reconstruction performance is inferior to the cases where the partitions are chosen adaptively based on the available data. Because of the high computational complexity of the available adaptive partitioning methods, there are very limited and usually small scale applications where data dependent non-uniform partitions are used.
In this work, a new data dependent recursive reconstruction algorithm is proposed for robust and efficient estimation of the unknowrs. In this algorithm, the parameteriz ation of the space of unknowns are performed by using an appropriate wavelet basis for the application at hand. The algorithm recursively updates the solution in an increasingly larger dimensional subspace whose basis vectors are chosen as a subset of the wavelet basis. Specifically which basis vectors should be used at each step depends on the available data. Robust criterions on how to chose the basis vectors at each iteration, and when to stop the iterations are given.

\section{RECURSIVE RECONSTRUCTION ALGORITHM}

In many physical data acquisition schemes, the measurements are related to the unknowns as in the following general form:

$$
y=K x+v
$$

where $y$ is the $m$-dimensional vector of available measurement data, $K$ is the measurement kernel or operator, $x$ is the unknown and $v$ is additive measurement noise with known mean and covariance. In this operator form of the measurement relation $x$ can be a function of one or more variables, or a vector or matrix with entries as the samples of the unknown function. A robust reconstruction can be obtained based on a detailed analysis of the measurement kernel, which requires significant amount of computation. Hence, usually applications of this type of algorithms are usually limited to the small scale problems $[5,6]$.

In the following, an efficient reconstruction algorithm will be developed for robust and accurate estimation of the unknowns. In this approach, an optimal subspace of the domain of $x$ will be searched such that least-squares inversion within this subspace provides a satisfactory reconstruction. For this purpose, a properly chosen wavelet basis can be used. The search for the optimal subspace will be performed in steps of increasing dimensions with the addition of new basis components to the existing ones in the previously formed subspace. It is important to find an efficient way of determining the order in which the basis components should be used. In one of the possibilities, the basis components $\phi_{i}$ are ordered such that $\left|{ }^{H} K \phi_{i}\right|$ is decreasing with $i$ increasing. This ordering can be motivated by 
writing the measurement relation as:

$$
y \approx K \sum_{i=1}^{N} \phi_{i} \alpha_{i}
$$

where $\sum_{i=1}^{n} \phi_{i} \alpha_{i}$ is the decomposition of the $x$ on the first $n$ basis components. As it can be seen from this form

$$
b_{i}=K \phi_{i}
$$

plays an important role, where Eqn. 2.2 can be interpreted as the decomposition of $y$ onto the possibly non-orthogonal vectors $b_{i}$. Therefore, for larger values of $\left|y^{H} b_{i}\right|$, one can expect to estimate $\alpha_{i}$ more reliably. Since, for a wavelet basis there exist fast decomposition algorithms, $b_{i}$ can be obtained efficiently from the wavelet decomposition of the rows of the kernel $K[7]$. Although this ordering scheme of the basis vectors is computationally very efficient one, because of the non-orthogonality of the basis components, it may fail to provide the optimal subset of the basis components which has the largest projection energy of $\boldsymbol{y}$. However, the optimal subset of the basis components can be obtained by using the matching-pursuit algorithm. In this approach, the first basis component is chosen as the one which maximizes $\left|y^{H} K \phi_{i}\right|$. Then at step $n$ of the recursions the optimal set of basis components is updated by adding the basis vector which has the largest absolute inner product with the residual measurement vector, i.e.,

$$
b_{n+1}=\arg \max _{b_{i}} \quad\left|\left(\boldsymbol{y}-\boldsymbol{y}_{n}\right)^{H} \boldsymbol{b}_{i}\right|
$$

where $y_{n}$ is the optimal estimate of the measurement by using $n$ basis components.

These two approaches of ordering the basis components will be used in conjunction with the recursive algorithm derived in the rest of this section providing the least-squares solution of $x$ in the obtained sequence of enlarging subspaces. First define the decomposition of $x$ onto the first $n$ basis components to be:

$$
x_{n}=\sum_{i=1}^{n} \phi_{i} \alpha_{i}
$$

therefore, $n^{\text {th }}$ order estimate of $\boldsymbol{x}$ requires the estimation of $\alpha_{n}=\left[\alpha_{1} \ldots \alpha_{n}\right]^{T}$, which can be obtained as the solution to:

$$
\min _{\alpha_{n}} \quad\left\|y-B_{n} \alpha_{n}\right\|^{2}+\mu\left\|\alpha_{n}\right\|^{2}
$$

where $B_{n}=\left[b_{1} \ldots b_{n}\right]$, and $\mu$ is the ridge-regularization constant which can be set to zero to obtain the least-squares solution [2]. Closed form solution of this optimization problem is:

$$
\left.\hat{\boldsymbol{\alpha}}_{n}=\widehat{(\boldsymbol{B}}{ }_{n}^{H} \boldsymbol{B}{ }_{n}+\mu \boldsymbol{I}\right)^{-1} \boldsymbol{B}_{n}^{H} \boldsymbol{y} \text {. }
$$

Since the optimal number of basis components to be used is not known a priori, estimates for $\hat{\alpha}_{n}$ for various values $n$ should be obtained in the search for the right number of basis components. If the above closed form solution is used for the estimates, a lot of computation should be done for the required matrix inversions, which makes its use limited to small scale problems. Fortunately, there is an efficient way of updating the inverse matrix $\left(B_{n}^{H} B{ }_{n}+\mu I\right)^{-1}$ for two consecutive values of $n$ providing efficiency in the required computations. The following recursive algorithm is based on this idea of updating the inverse matrix in unit increments of $n$ beginning with 1 . At each step of the algorithm, a computational count is also given. At step 1 compute:

$$
\begin{aligned}
H_{1} & =\left(B_{1}^{H} B_{1}+\mu I\right)^{-1} \\
h_{1} & =b_{1}^{H} y \\
\hat{\alpha}_{1} & =H_{1} h_{1}
\end{aligned}
$$

where for an $m$-dimensional $y$, at Eqn. $2.8 m$ multiplications, $m$ additions and 1 division, at Eqn. $2.9 m$ multiplications, $m-1$ additions, and at Eqn. 2.101 multiplication are required. The general step of the algorithm which updates $H_{i}$ makes use of the following matrix inversion result:

$$
\left[\begin{array}{cc}
R & r \\
r^{H} & \rho
\end{array}\right]^{-1}=\left[\begin{array}{cc}
Q & q \\
q^{H} & \kappa
\end{array}\right]
$$

where $\boldsymbol{R}$ is an invertible matrix, $\boldsymbol{r}$ is a vector and $\rho$ is a scalar and:

$$
\begin{aligned}
\kappa & =\frac{1}{\rho-r^{H} R^{-1} r} \\
q & =-\kappa R^{-1} r \\
Q & =R^{-1}-R^{-1} r q^{H}
\end{aligned}
$$

The validity of this useful inversion result can be checked by multiplying the original matrix and the proposed inverse of it. This formula is the backbone of the general step of the recursions. However, in order to further reduce the computations, in the recursions some other intermediate variables are used. The general step of the recursion is the update from $n$ to $n+1$, given by:

$$
\begin{aligned}
\boldsymbol{\theta}_{n+1} & =\boldsymbol{B}_{n}^{H} b_{n+1} \\
\gamma_{n+1} & =H_{n} \boldsymbol{\theta}_{n+1} \\
\beta_{n+1} & =\frac{1}{b_{n+1}^{H} b_{n+1}+\mu-\theta_{n+1} \gamma_{n+1}} \\
\eta_{n+1} & =-\gamma_{n+1} \beta_{n+1} \\
H_{n+1} & =\left[\begin{array}{cc}
H_{n}-\gamma_{n+1} \eta_{n+1}^{H} & \eta_{n+1}^{H} \\
\eta_{n+1}^{H} & \beta_{n+1}
\end{array}\right] \\
\epsilon_{n+1} & =\beta_{n+1}\left(b_{n+1}^{H} y\right) \\
\nu_{n+1} & =\left(\theta_{n+1}^{H} \hat{\alpha}_{n}\right) \beta_{n+1}-\epsilon_{n+1} \\
\hat{\alpha}_{n+1} & =\left[\begin{array}{c}
\hat{\alpha}{ }_{n}+\nu_{n+1} \gamma_{n+1} \\
-\nu_{n+1}
\end{array}\right] .
\end{aligned}
$$

In this general step of the recursion $2 n^{2}+n(m+4)+2 m+2$ multiplications, 1 division, and $2 n^{2}+n(m+1)+2 m-1$ additions are required to compute. Since a multiplication requires more computation than a summation, the computational complexity is determined by the number of multiplications. The total number of multiplications required to compute $\hat{\boldsymbol{\alpha}}_{n}$ can be found by counting all the multiplications required by the previous updates which adds up to $2 n^{3} / 3+n^{2}(m / 2+2)+(7 / 3+5 / 2 m) n-3 m-5$ which is $O\left(m n^{2}\right)$ for $m>n$. Note that direct use of Eqn. 2.7 requires $O\left(m n^{2}\right)$ multiplications for each $n$. Therefore, the 
computational saving of the recursive algorithm over the direct solution is significant. Also, the recursive algorithm provides estimates $\hat{\alpha}_{n}$ at each step of the recursion making it possible to easily implement criterions to stop the iteration. One important quantity that is helpful in the decision to stop the iterations is the measurement fit error:

$$
e(n)=\left\|y-B_{n} \hat{\alpha}_{n}\right\|,
$$

which is a decreasing function of $n$. One commonly used criterion stops the iterations when $e(n)$ is either small enough or reaches a plateau region following a fast decrease. Although, this criterion provides reasonable reconstructions, it usually under estimates the optimal value of $n$. Another stop criterion is based on the squared magnitude of the estimate at each step, which can be computed along with the iterations using the fact that $\left\|\boldsymbol{x}_{n}\right\|^{2}=\left\|\boldsymbol{\alpha}_{n}\right\|^{2}$. One way of terminating the iterations base $\mathrm{X}$ on this quantity is given in the next section.

\section{SIMULATIONS}

In this section, the two different methods of ordering the basis components in the recursive reconstruction algorithm are compared with each other. The ordering scheme based on matching pursuit algorithm is referred to as method-1. Method-2 is used to refer to the scheme where the basis components are ordered such that $\left|y^{K} K \phi_{i}\right|$ is decreasing with $i$ increasing. These two methods provide the same result if $b_{i}$ in Eqn. 2.3 form an orthogonal set. Otherwise, it is expected that the method-1 provides better estimates at a higher computational cost than the method- 2 does. For this investigation, both methods are used in a medium sized reconstruction problem with 256 1-dimensional unknowns and 500 measurements. In the first simulation example, additive noise is chosen to be i.i.d. Gaussian with variance 0.01 . The measurement kernel is chosen such that the energy of its columns decreases rapidly as the column index gets larger. This type of rapid decrease is a common case in remote sensing applications where the domain of unknowns is partitioned with a uniform grid.

In this example Haar basis is chosen to be the wavelet basis for the domain of the unknowns. The regularization parameter of the reconstruction algorithm, $\mu$, is set to 0 . In Fig. 1.a the measurement fit errors of both methods are shown. It can be seen that the measurement fit error decreases faster for method-1. This is a natural consequence of the matching pursuit ordering used in method-1. The magnitudes of the estimated unknown $x_{i}$ shown in Fig. 1.b is used to determine the number of basis components to be used in the reconstruction. In this simulation, based on Figures $1 . a$ and $1 . b, 20$ and 10 basis components can be chosen for methods L and 2 respectively. It is important to note that when the regularization parameter $\mu$ is set to zero, a rapid increase in Fig. 1.b is an indication of what is usually referred to as "noise fitting", which should be avoided in an acceptable estimation. One reason behind the choice of this particular simulation example is to show that, although it is commonly used, Fig. 1.a is not so informative in the determination of when to stop the iterations in a recursive reconstruction algorithm.
The corresponding reconstructions are shown in Fig. 2.a together with the actual unknown vector $\boldsymbol{x}$. In Fig. 2.b the actual noisy measurement and the its close fits provided by method- 1 and 2 are shown. As expected, method- 1 outperforms method- 2 in this simulation. Note that, since the energy of the columns of the measurement kernel $K$ decreases with the column index, in the reconstructions higher resolution components are located near the beginning, and lower resolution components are located near the end as expected. This has been achieved automatically by the data dependent orderings of the wavelet basis by the both methods used.

In another simulation where the same measurement kernel is used but the measurement noise variance is chosen to be 100 times larger is shown in Figures 3 and 4. Based on similar arguments, this time 13 basis components are chosen for method- 1 and 10 basis components are chosen for method-2. Also in this case method-1 outperforms method2 , but the margin is not as large as the previous case shown in Figures 1 and 2.

The performance of the proposed recursive reconstruction algorithm has been tested in many other cases. In general high quality reconstructions have been obtained. Especially in cases of low SNR in the measurements, the obtained results have been superior to those of other reconstruction approaches.

\section{CONCLUSIONS}

A new data dependent recursive reconstruction algorithm is proposed for robust and efficient estimation of the unknowns. The algorithm recursively updates the solution in an increasingly larger dimensional subspace whose basis vectors are chosen as a subset of the wavelet basis. Robust criterions on how to chose the basis vectors at each iteration, and when to stop the iterations are given. It is demonstrated that very satisfactory results are obtained by using the proposed algorithm.

\section{REFERENCES}

[1] A. N. Tikhonov and V. Y. Arsenin, "Solutions of IllPosed Problems," New York: Wiley, 1977.

[2] A. E. Hoerl and R. W. Kennard, "Ridge regression," American J. Math. Management Sci. vol. 1, pp. 5-83, 1981.

[3] O. Arrkan and B. Baygün, "Least squares signal reconstruction under normalized autocorrelation constraints," , vol. 29, pp. 519-538, 1994.

[4] Golub, G. H. and Van Loan, C. F., Matrix Computations, $2^{\text {nd }}$ ed., Baltimore: John Hoppkins Univ. Press., 1989.

[5] L. G. Chambers, Integral Equations: A Short Course, London: Inter. Textbook Co., 1976.

[6] L. M. Delves, and J. L. Mohamed, Computational Methods for Integral Equations, Cambridge: Cambridge University Press, 1985.

[7] 1. Daubechies, Ten Lectures on Wavelets, CBMS-NFS Regional Conference Series in Applied Mathematics, Vermont: Capital City Press, 1992. 

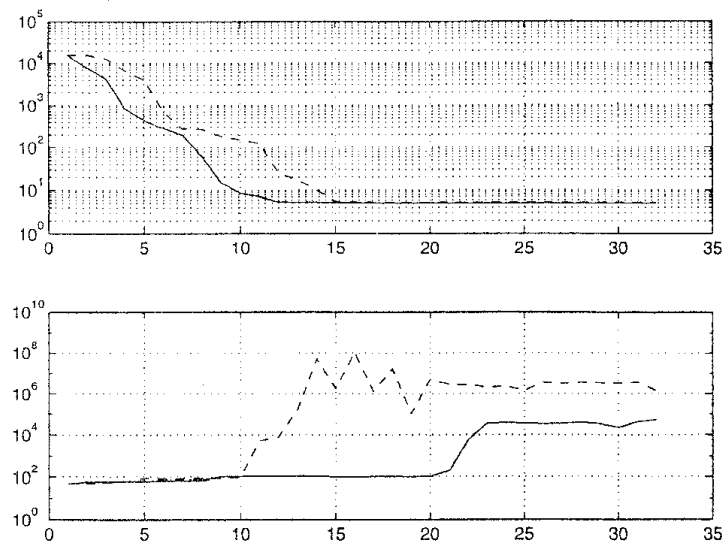

Figure 1: a. Plot of magnitude of measurement fit errors, method-1:', method-2: ', , (top). b. Squared magnitude of the estimate versus number of basis components used, method-1:'-', method-2:'-' .
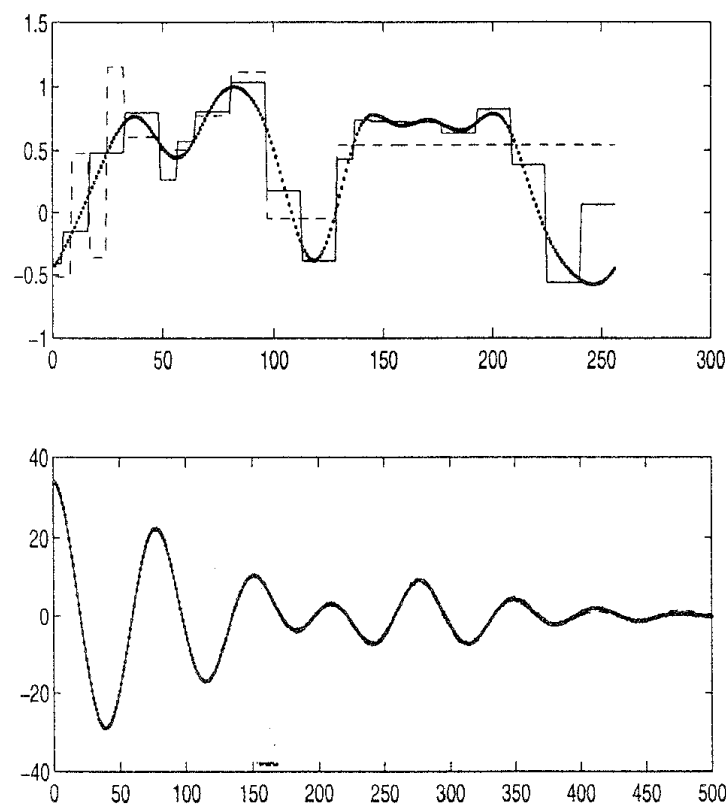

Figure 2: a. Actual (shown as: ' $\because$ ) and recovered $x$., (method-1:-', method-2: $\left.:^{6}-1\right),($ top $)$. b. Fit to the actual measurement.
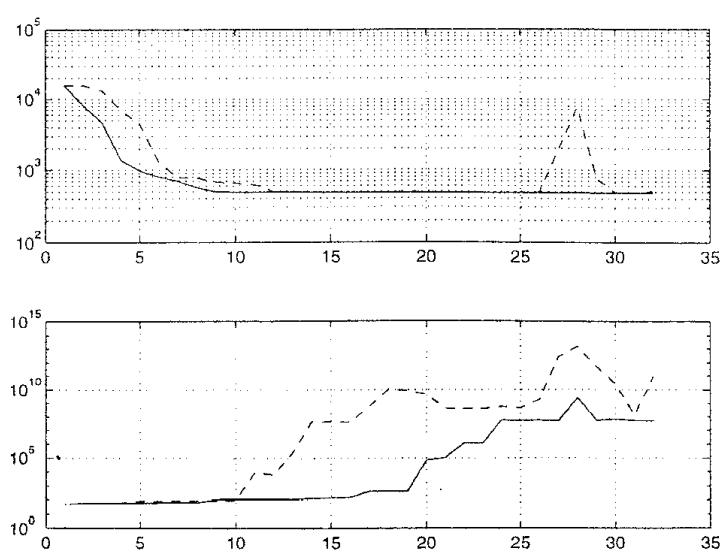

Figure 3: a. Plot of magnitude of measurement fit errors, method-1:'-', method-2: - ', (top). b. Squared magnitude of the estimate versus number of basis components used in a lower SNR case, method-1:'-', method-2:' ${ }^{6}$.
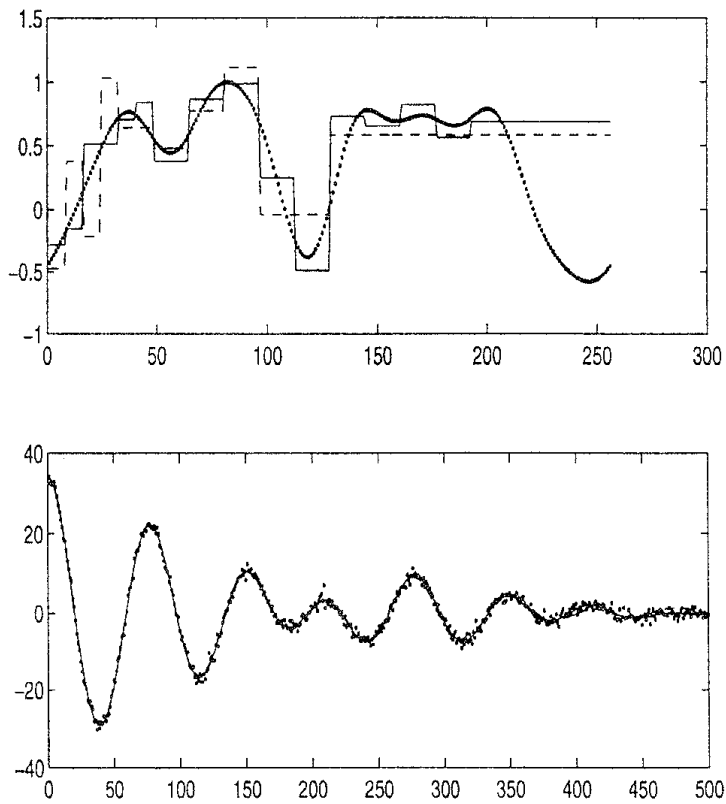

Figure 4: a. Actual (shown as: ' ') and recovered $x$., (method-1:-', method-2:-' ),(top). b. Fit to the actual measurement in a lower SNR case (actual: '. ', method-1:'-', method $-2:^{6}-$ ) 